\title{
An Investigation of the Dimensional Changes of Polymer Mixture Tablets Containing a Soluble Drug, Using an Image Analysis Method. Influence of These Characteristics on Drug Release and Its Mechanism
}

\author{
Manuel Efentakis*, Despina Tavoulari \\ Department of Pharmaceutical Technology, Faculty of Pharmacy, University of Athens, Athens, Greece. \\ Email: "efentakis@pharm.uoa.gr
}

Received January $5^{\text {th }}, 2012$; revised February $15^{\text {th }}, 2012$; accepted March $8^{\text {th }}, 2012$

\begin{abstract}
The properties and characteristics of the polymer used for the preparation of matrix drug delivery systems considerably influence their performance and the extent of drug release and its mechanism. The objective of this research was to examine the dimensional changes, and gel evolution of polymer matrices consisting of three different polymers Polyox (POL), sodium alginate (SA) (hydrophilic) and Ethocel (ET) (hydrophobic), using an image analysis method. Furthermore to explore how these changes influence the release rate of a soluble drug namely, venlafaxine (V). All tablets displayed marked dimensional expansion and gel growth particularly those consisting of two hydrophilic polymers Polyox/sodium alginate (POL/SA/V) compared to those consisting of the hydrophilic/hydrophobic Polyox/Ethocel (POL/ET/V). Similarly the thickness of the gel layer in POL/SA/V matrices increased considerably with time up to 8 hours. In general our findings show that the POL/SA/V matrices, due to their thicker gel layer produced a more effective barrier which results in a more pronounced sustained release delivery. This accounts for the slower and smaller overall drug release observed with the POL/SA/V matrices compared to those containing POL/ET/V and indicates that the formation of a thick and durable gel barrier is a characteristic necessary for the preparation of sustained drug release systems. Moreover the solubility of venlafaxine in combination with the polymer's properties appears to play an important role on the extent of drug release and the release mechanism. Overall the polymer mixtures examined comprise a useful and promising combination of materials for the development and manufacture of sustained release preparations based on these polymers.
\end{abstract}

Keywords: Polymer Mixture; Drug Release; Image Analysis; Dimensional Changes

\section{Introduction}

Polymeric materials and predominantly hydrogels are appropriate carriers for drug delivery due to their ability to influence drug release from systems based on these materials. Drug release from hydrogels is known to be affected by the degree of dimensional expansion/swelling and the properties of the gels that are formed.

Since only a few investigations of the behaviour of polymer mixtures and particularly their effect on drug release have been published [1-4] the aim of this study was to examine mixtures of three different polymers, Polyox[polyethylene oxide] with sodium alginate (both hydrophilic) or Ethocel [ethylcellulose] (hydrophobic), which could potentially be used as carriers for the preparation of sustained release dosage forms. Venlafaxine a

"Corresponding author. soluble drug was used as model compound.

Polyethylene oxide polymers are hydrophilic, swellable, uncross linked, non anionic polymers, soluble in water with a variety of molecular weights ranging from of 100,000 to $8,000,000$. Since these polymers are non anionic materials no interactions with drugs are expected. Recently they have been used in controlled drug-delivery systems [5]

Sodium alginate is the sodium salt of the natural hydrophilic polysaccharide of $\beta$-D-mannuronic and a-Lguluronic acids and is derived from seaweed. It forms a gel in the presence of aqueous fluids and this has provoked considerable interest in its use in drug delivery systems. The hydration characteristics of the polymer and the physical properties of the hydrated gel may modify drug release from alginate-based preparations [6-8]. Alginates are available in low, medium and high viscos- 
ity grades by suppliers. The different viscosity grades can give rise to significant changes in drug release from alginate preparations. The release of a water soluble drug from an alginate tablet depends on swelling and will be mainly by diffusion [7].

Ethylcellulose is an inert, hydrophobic polymer, insoluble in water that has been widely used in a number of dosage forms including as a matrix for sustained release dosage forms [9].

The infiltration of liquid into a matrix and particularly liquid uptake/swelling are important characteristics since both play a significant role on sustained release delivery from hydrophilic systems. Swelling is a complicated phenomenon governed by polymer stress relaxation, swelling and eventually liquid diffusion. The liquid penetrates into the polymer mass and when its concentration reaches a certain level the polymer swells and a gelatinous viscous mass (gel layer) is formed at the surface. The formed gel layer and its thickness [10-12], significantly affect the performance of the system, in particular the rate and mechanism of drug release [12].

Several methods have been used to determine axial and radial expansion and the measurement of tablet dimensions. The techniques used, include removal of the preparation and weighing, occasional photography of the tablet against a calibrated graticule and recently image analysis $[13,14]$. The image analysis method permits accurate measurement of tablet dimensions with a noninvasive technique and this combined with the gel layer evolution/changes during the swelling process offers an overall evaluation of the material employed.

The aim of this study was to examine dimensional changes/swelling, the mode in which these changes influenced dimensional changes in an axial and radial direction during swelling, as well as the formation and development of the gel layer, in tablets made of Polyox with sodium alginate or Ethocel since these characteristics have significant effects on tablet's structure and regulate the overall performance of oral drug delivery preparations. Finally we aimed to evaluate and determined the effects of those changes on drug release and release mechanism.

\section{Experimental}

\subsection{Materials}

The following chemicals were obtained from commercial suppliers and used as received:

Polyethylene oxide [Polyox of $4 \times 10^{6} \mathrm{MW}$ ] (Union Carbide, CT), sodium alginate high viscosity (Sigma chemical, Co), ethylcellulose [Ethocel, 50cps, Dow Chemicals, PA] and magnesium stearate (BDH, Dorset, UK). Venlafaxine HCI (Gador Lab. SA) was used as model drug.

\subsection{Tablet Preparation}

Pure polymer tablets, and polymer mixture tablets with Polyox and sodium alginate or Ethocel, containing $50 \mathrm{mg}$ velafaxine, $475 \mathrm{mg}$ weight, with $10 \mathrm{~mm}$ diameter and 5 $\mathrm{mm}$ height, were prepared. The mixing of Polyox with sodium alginate or Ethocel venlafaxine and $1 \%$ magnesium stearate was carried out in a Turbula T2F (Willy A. Bachofen AG. Basel, Switzerland), laboratory mixer for $10 \mathrm{~min}$. The powders were compressed to tablets using a Carver laboratory hydraulic press, (Fred S. Carver, Inc., Menomonce Falls, W), to a crushing strength of $16-18$ $\mathrm{kg}$, (measured in the Erweka TBH 28 hardness tester). This strength is commonly used in sustained release tablets. Their friability was found in the region of $1 \%$ and determined using the Roche friability tester (Erweka TA).

\subsection{Optical Image Analysis}

The method we used was similar to the method described in a previous study $[13,15]$. The recorded images were collected and analyzed with a Leica image analysis system (Leica Q $5001 \mathrm{~W}$ ). A video camera (JVC TK-C11381, Japan) was fitted with a zoom lens (Century Precision Optics AD-5870, USA) and connected to a monitor. The light system consisted of a fluorescent tube fitted under the beaker. The beaker was covered to prevent external light. The tablet was held on a pin and placed in a dissolution beaker (of a dissolution apparatus) with $900 \mathrm{ml}$ of distilled water at $37^{\circ} \mathrm{C} \pm 0.5^{\circ} \mathrm{C}$ at $50 \mathrm{rpm}$ stirring. The tab- let was mounted in a vertical or horizontal direction to allow the observation of swelling in the axial or radial direction. The beaker was removed, at predetermined time intervals from the dissolution apparatus and was transferred to the optical image set up and photographed by means of a video camera, to observe the expansion procedure and the gel layer changes. At defined time intervals, the image was measured and the height (axial) or diameter (radial) as well as gel layer changes were recorded. Results reported are averages for three different tablets. The swelling values of axial, radial and gel layer were obtained by calibration of the obtained image; this allowed us to correlate the area of a pixel with physical dimensions. The dimensional scale was calibrated from the known tablet size and measurement of the image obtained at $t=0$. In the digital image technique, the image is converted into a number of points called pixels. Each pixel has a gray level from 0 (blanc) to 255 (white) representing the scattered light intensity. The characterization of water content was based on the variation of relative gray level intensity inside the tablet mass. The inten- 
sity of gelled region is a function of its hydration. A gray level intension of zero corresponds to a dry region in the matrix. In a typical optical image the gel layer appears as a white ring, due to the scattering of light by the hydrated polymer mass. The core does not permit the transmission of light and as a result it appears dark. The liquid also appears dark due to the lack of scattering. The two boundaries of the gel layer are clearly indicated by the sharp bright ring around the tablet, as it is shown in the photos referred below in the Results and Discursion sections.

\subsection{Dissolution Studies}

The dissolution studies were carried out according to the procedure described in USP using a dissolution tester (Pharmatest, Hainburg, Germany), paddle method, in 900 $\mathrm{mL}$ distilled water at $37^{\circ} \mathrm{C} \pm 0.5^{\circ} \mathrm{C}$ with the stirring at 50 rpm. At a selected time interval samples of venlafaxine HCI were withdrawn, filtered and analyzed at $225 \mathrm{~nm}$ using a Perkin Elmer UV spectrophotometer (Norwalk, CT, USA).

An equivalent volume of temperature-equilibrated fluid was replaced into the dissolution bath following the removal of every sample. The data represent the mean values of at least three separate experiments and the mean cumulative percentage of drug calculated ( \pm standard deviation) and plotted against time.

\section{Results and Discussion}

\subsection{Radial/Axial Dimensional Changes}

As mentioned above the polymers used in this study were the hydrophilic sodium alginate and Polyox and the hydrophobic Ethocel. The characteristics determined for the polymer tablets were: 1) the diameter (radial direction) 2) the height-thickness (axial direction) 3) the surface 4) the axial gel expansion and 5) the radial gel expansion.

Soon after the tablet came in touch with the liquid, a rapid and substantial expansion (swelling) and increase in size was exhibited in both axial and radial dimensions. Figure 1 shows the radial(DIAM) and axial(TH) expansion dimensional changes (normalized to the dry tablet) with time up to $8 \mathrm{~h}$ for tablets comprising either of pure polymers (Figure 1(a)) from mixtures of Polyox with sodium alginate or Ethocel, (Figures 1(b) and (c)). The pure Polyox and sodium alginate tablets along with the polymer mixture tablets underwent fast hydration caused by rapid liquid penetration into the polymer mass with the exception of pure Ethocel tablets which displayed insignificant expansion due to the hydrophobic nature of the polymer (Figures 2 and $\mathbf{3}$ ).

In these photos typical images of the pure tablets (Polyox, Ethocel and sodium alginate) and tablet mix-
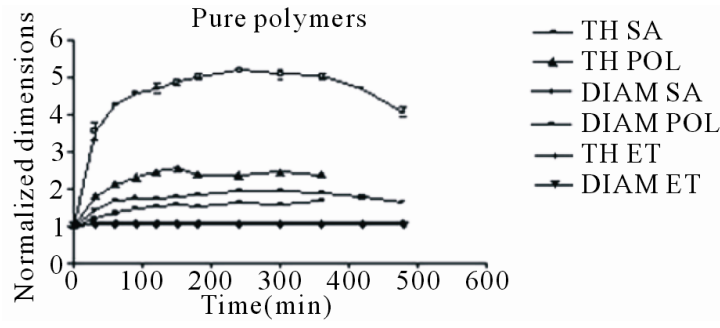

(a)
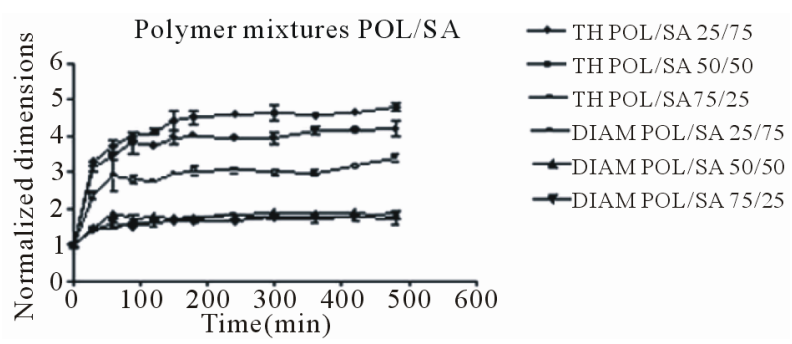

(b)
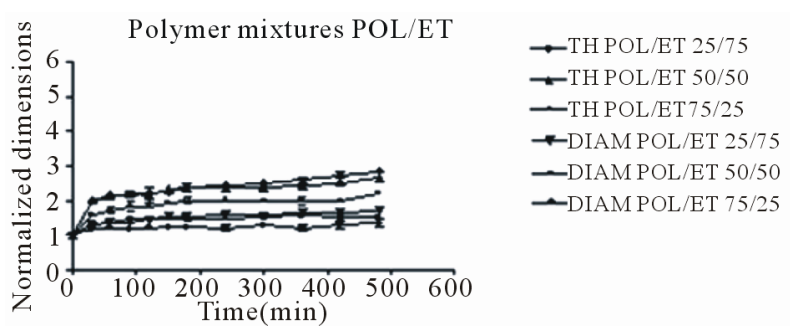

(c)

Figure 1. Radial (DIAM) and Axial (TH) dimensional changes for pure polymers and polymer mixtures. Each point represents the mean value of the three samples and error bars show \pm SD.
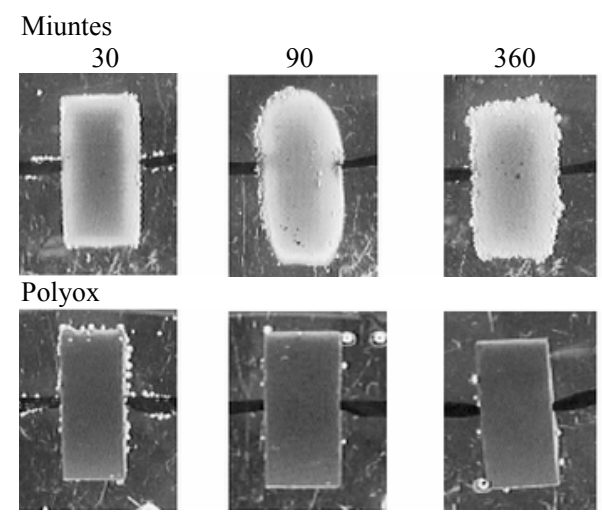

Ethocel
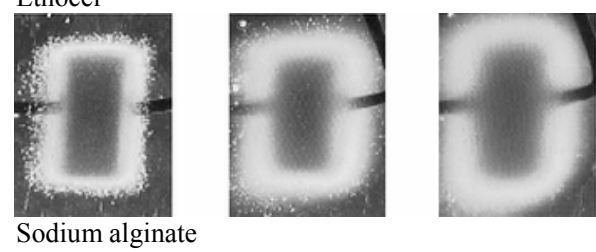

Figure 2. Morphological changes of pure Polyox, Ethocel and sodium alginate tablets after 30,90 and $360 \mathrm{~min}$. 
Miuntes
30

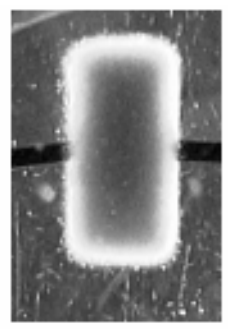

90

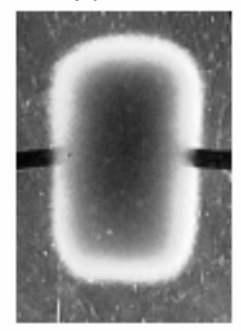

POL/SA/VENLAFAXINE 50/50

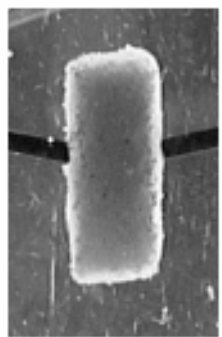

POL/ET/VENLAFAXINE 50/50
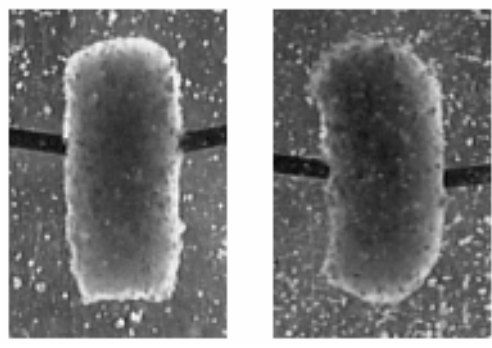

Figure 3. Morphological changes of polymer mixture matrices POL/SA/V and POL/ET/V after 30, 90 and $360 \mathrm{~min}$ containing venlafaxine.

tures (Polyox/sodium alginate and Polyox/Ethocel 50\%/ $50 \% \mathrm{w} / \mathrm{w}$ containing $50 \mathrm{mg}$ of venlafaxine) illustrate the changes in their radial and axial planes during the swelling process after 30, 90 and 360 minutes. In these images different characteristics are clearly visible: 1 ) an increase in the diameter and the thickness-height of the tablet with time, 2) evolution of the gel layer with time and 3) the change in the size of the dry core as more of the polymer becomes hydrated. These effects are due to hydration of the tablet and diffusion of the medium into the polymer mass followed by the swelling (expansion).

In less than $90 \mathrm{~min}$, the tablets exhibited considerable dimensional expansion. Anisotropic swelling behaviour was observed with preferential expansion in the axial dimension relative to the radial dimension for all formulations, in accordance with earlier studies $[13,14]$.

Also it is evident that the dimensional changes of the tablets with time appeared to be dependent on polymer properties. In Figure 1(a) the dimensional changes in pure polymer tablets are illustrated up to $8 \mathrm{~h}$, soon after the tablets changed shape and became deformed making further accurate measurements difficult.

Sodium alginate tablets exhibited the greatest expansion followed by Polyox and Ethocel. As seen in all cases the Polyox/sodium alginate (POL/SA), tablets, (Figure 1(b)), composed of the hydrophilic materials, exhibited the greatest expansion, in both axial and radial planes, compared to Polyox/Ethocel (POL/ET) tablets, (Figure 1(c)), composed of hydrophilic and hydrophobic materi- als. This was apparently due to the presence of two hydrophilic polymers in the POL/SA tablets resulting in a greater sorption of liquid by these polymers, (Figures 1, 2).

Moreover as the amount of sodium alginate increased a greater expansion in the tablet size was observed. On the contrary in POL/ET tablets, a smaller increase in tablet size was observed since Ethocel is hydrophobic and therefore exhibits negligible swelling, (Figure 1(a)).

Both mixture tablets continued to expand throughout the duration of the 8 hour and as mentioned above the POL/SA tablets exhibited the greatest swelling rate and overall greatest expansion.

The maximum axial increase was $4.65,4.15$ and 3.40 folds for the tablets containing 75, 50 and 25 percent sodium alginate (POL/SA) respectively, (Figure 1(b)). For the POL/ET tablets the maximum increase was 2.85, 2.45 and 2.10 folds for those containing 25, 50 and 75 percent Ethocel (Figure 1(c)). Radial expansion followed an analogous trend but with smaller increases for both mixtures. It is obvious, that the decrease in tablet expansion was associated with an increase in Ethocel content.

With regards to polymer mixture matrices containing venlafaxine, it appears that their expansion was a little greater and more rapid probably due to the presence of the very soluble drug $(1000 \mathrm{mg} / \mathrm{ml}$ in water), which facilitates liquid penetration, however after $6 \mathrm{~h}$ a small decrease was observed, (Figure 4). The maximum axial increase of the POL/SA/V matrices was 5.20, 4.60 and 4.0 folds for those containing 75,50 and 25 percent sodium alginate respectively, (Figure 4(a)), while for Ethocel matrices(POL/ET/V) the increase was 2.90, 2.55 and 2.15 folds respectively (Figure 4(b)). Again radial expansion displayed an analogous trend but with smaller increases. A noted difference in the venlafaxine matrices was that maximum expansion was observed at $6 \mathrm{~h}$ and then a minor decrease was observed, while in the tablets without drug dimensional expansion continued until the end of the experiment $(8 \mathrm{~h})$. This could probably be attributed to the dissolution of venlafaxine and as a result of this shirking of the matrix was observed.

In all cases POL/SA tablets with or without drug demonstrated the greatest increase whereas within the same time period POL/ET tablets displayed the lowest increase, (Figures 2 and 3) The POL/SA (25\%/75\%) mixture exhibited the highest increase and POL/ET (25\%/ $75 \%$ ) the lowest.

Visual observations revealed in POL/ET tablets that after the $5^{\text {th }}$ hour particles, possibly comprised of Ethocel (particularly from tablets $25 \% / 75 \%$ mixtures), detached from the surface of the tablet causing increased deterioration and shrinking of the tablet. 
An Investigation of the Dimensional Changes of Polymer Mixture Tablets Containing a Soluble Drug, Using an Image Analysis Method. Influence of These Characteristics on Drug Release and Its Mechanism

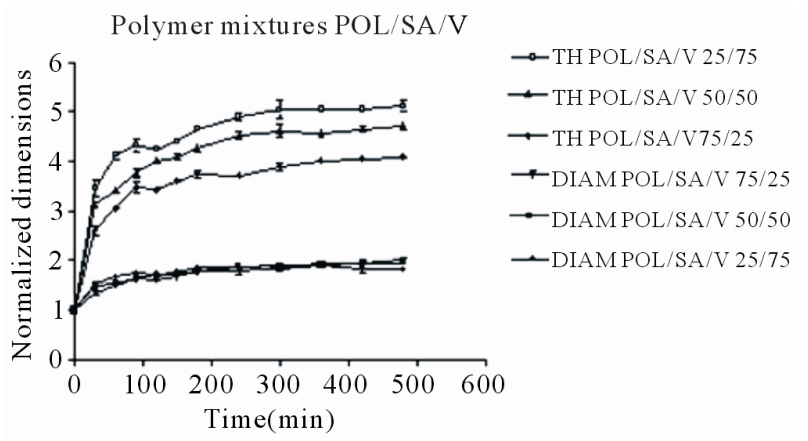

(a)

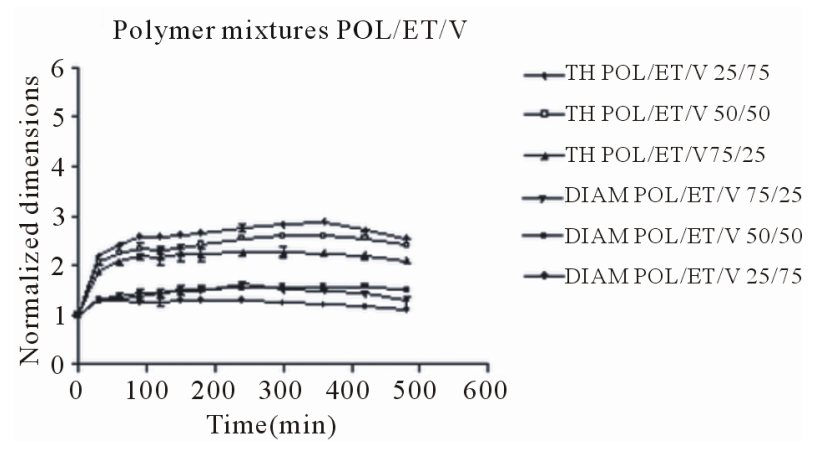

(b)

Figure 4. Radial (DIAM) and Axial (TH) dimensional changes for polymer mixtures matrices POL/SA/V and POL/ $E T / V$, containing venlafaxine. Each point represents the mean value of the three samples and error bars show \pm SD.

Measurement of the diameter and thickness of the expanded tablet allowed the calculation of the corresponding total surface using the formula, Area $=2 \mathrm{r} \pi \cdot(\mathrm{r}+\mathrm{h})$. The results were normalized to the surface of a dry tablet and are shown in Figure 5. As expected both POL/SA and POL/ET tablets, demonstrated a comparable surface increase as a result of their analogous axial and radial expansion. Therefore the greatest increase was exhibited by the POL/SA $25 \% / 75 \%$ mixture followed by the $50 / 50$ and $25 \% / 75 \%$ mixtures. In the POL/ET tablets the greatest increase was exhibited by the $75 \% / 25 \%$ POL/ET followed by the $50 / 50$ and $25 \% / 75 \%$ mixtures, (Figure 5(a)). A similar trend was also observed in the tablets containing venlafaxine (Figure 5(b)).

\subsection{Gel layer Changes}

In Figures 2 and $\mathbf{3}$ we can see that a viscous gel layer formed around the surface of the swelled tablets and an increase in the gel layer resulted in shrinking of the core. The thickness of the gel layer and its changes play a significant role and influence drug release from swellable delivery systems [12].

Hence it is important to study the development of the

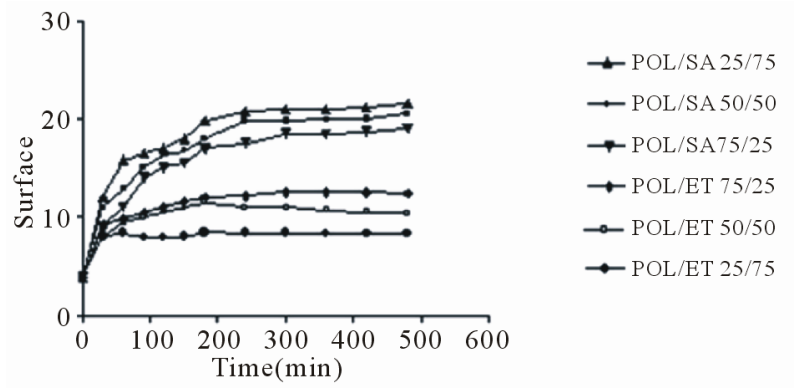

(a)

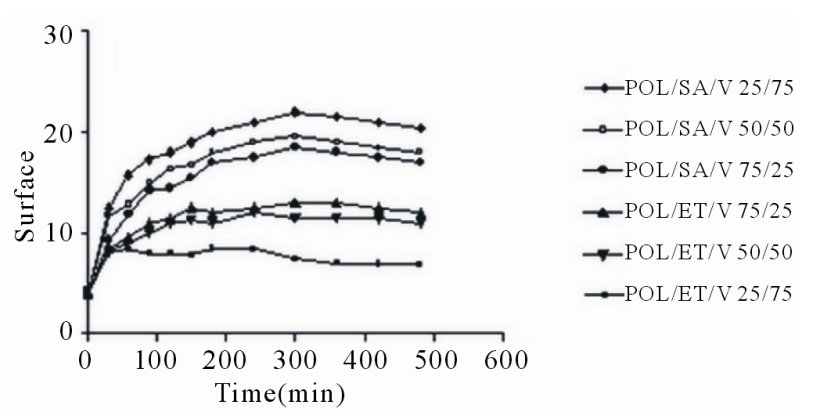

(b)

Figure 5. Surface changes of a) POL/SA, POL/ET; b) POL/ $\mathrm{SA} / \mathrm{V}, \mathrm{POL} / \mathrm{ET} / \mathrm{V}$ tablets vs. time during hydration. Each point represents the mean value of the three samples and error bars show \pm SD.

gel layer. Development changes are shown in Figure 6. The distance from the gel/core interface to the outer surface of the gel was taken as a measurement of the thickness of the layer. Average radial and axial gel thickness values are shown, since previous studies [12,14] have suggested that, the use of average radial and axial gel thickness values achieves better precision and evaluation. Figure 6 shows the development and changes in gel layer thickness up to $8 \mathrm{~h}$ for all tablets examined. After that time the tablets were deformed making accurate measurement of gel layer thickness difficult.

As may be observed, polymer tablets without drug exhibited greater gel thickness than equivalent tablets with drug. In addition POL/SA tablets developed a thicker gel layer than the POL/ET tablets. This could be attributed, as mentioned above, to the presence of two hydrophilic polymers in the POL/SA mixture.

It is noteworthy that the increase in gel thickness was analogous to dimensional expansion as shown in Figure 3. Furthermore, it is clear that initially there was a rapid increase in gel thickness in the mixture tablets without venlafaxine up to $240 \mathrm{~min}$ and then, a plateau was observed until the end of experiment.

At the same time in matrices with drug apart from the smaller increase in the gel layer, a decrease was demonstrated in POL/SA/V matrices after $5 \mathrm{~h}$ and in POL/ET/V 


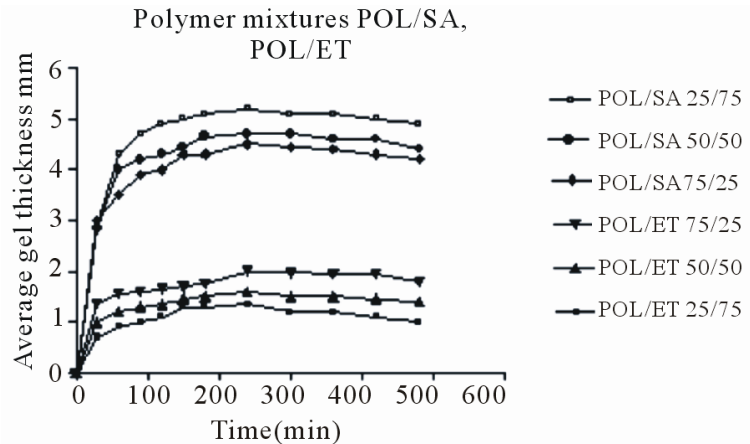

(a)

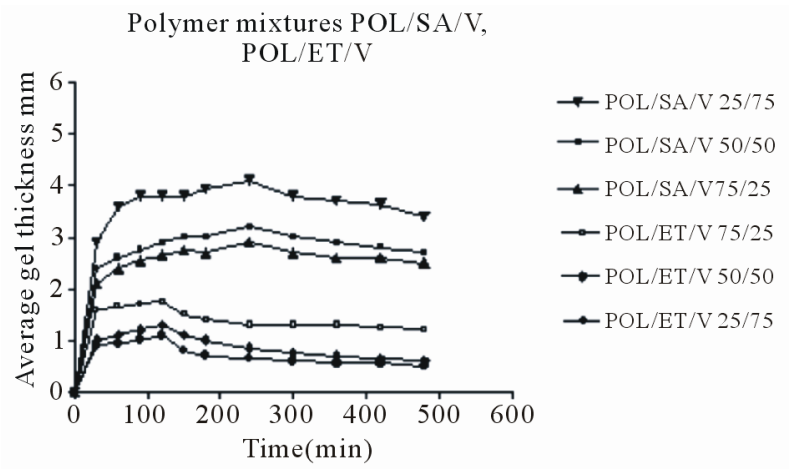

(b)

Figure 6. Average gel thickness growth vs. time; (a) POL/ SA, POL/ET; (b) POL/SA/V, POL/ET/V tablets during hydration. Each point represents the mean value of the three samples and error bars show \pm SD.

matrices after $2.5 \mathrm{~h}$. This could be attributed to the presence of the soluble drug which may affect the integrity of gel layer, reduce the entanglement of polymer chains and reduce its thickness. This was more intense in the mixtures containing higher concentrations of Ethocel.

The maximum increase in the POL/SA tablets was 5.2, 4.7 and $4.4 \mathrm{~mm}$ for $75 \%, 50 \%$ and $25 \%$ sodium alginate content respectively (Figure 6(a)). With the POL/ET tablets the maximum values were lower i.e. 2.1, 1.6 and $1.3 \mathrm{~mm}$ for $25 \%, 50 \%$ and $75 \%$ Ethocel, correspondingly. On the other hand for POL/SA/V matrices maximum increases were 4.0, 3.2 and $2.9 \mathrm{~mm}$, (Figure 6(b)), while for POL/ET/V matrices there were $1.8,1.3$ and $1.1 \mathrm{re}-$ spectively. These results demonstrate that the POL/SA mixtures produced a higher degree of swelling and gel growth compared to the equivalent POL/ET mixtures with or without drug.

\subsection{Polymer Effects on Drug Release}

In Figure 7, the release profiles from the prepared matrix tablets are illustrated.

As can be seen the POL/ET/V 25/75 preparation exhibited the highest release $80 \%$, followed by $50 / 50$

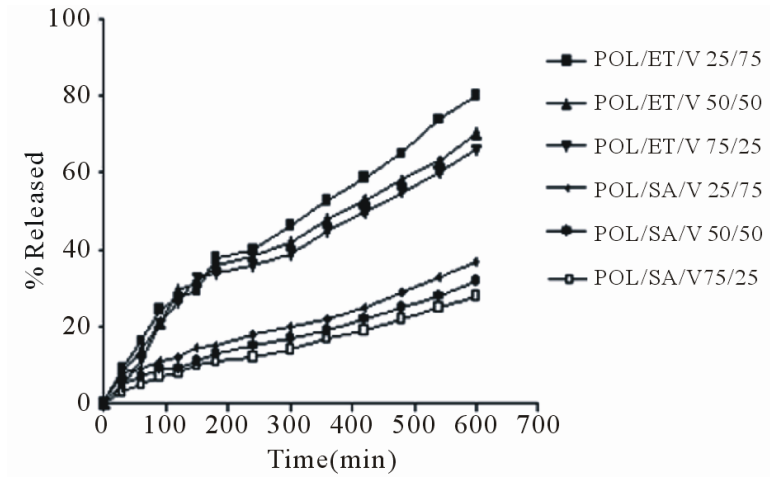

Figure 7. Vennlafaxine release profiles from POL/SA/V and POL/ET/V matrices.

preparation with $70 \%$ and $75 / 25$ with $65 \%$. In parallel $\mathrm{POL} / \mathrm{SA} / \mathrm{V}$ tablets displayed a much lower release more specifically $37 \%$ for $75 / 25$ preparation followed by $32 \%$ for the $50 / 50$ and $28 \%$ for the $25 / 75$ preparations.

It is obvious that drug release from $\mathrm{POL} / \mathrm{ET} / \mathrm{V}$ mixture matrices is greater than the corresponding $\mathrm{POL} / \mathrm{SA} / \mathrm{V}$ matrices and the reason is that the hydrophilic POL/SA mixtures produce a stronger barrier i.e. a thicker gel layer than POL/ET and as a consequence hinder the movement of drug molecules (Figure 6).

Drug release from a matrix tablet may be considered as a mass transport phenomenon and generally involves three different steps. Liquid penetration into the tablet is the first step followed by dissolution of the drug and finally its diffusion.

However the diffusion of drug molecules is affected by the surface area of the matrix exposed to the medium and particularly thickness of the gel layer. This was evident.

Figure 8, with the POL/SA/V matrices as their greater surface area and gel layer thickness resulted in the lowest overall drug release rate compared to the corresponding $\mathrm{POL} / \mathrm{ET} / \mathrm{V}$ matrices which displayed the highest release rate. The other preparations demonstrated intermediate values. These results clearly indicate that the surface area exposed and the gel thicknesses considerably affect the rate of drug release from these matrices.

In general these findings suggest that $\mathrm{POL} / \mathrm{SA} / \mathrm{V}$ matrices, particularly, with their thicker gel layer would produce a stronger and more effective barrier for sustained release delivery, in view of the fact that the diffusion path is enlarged. For this reason a slower drug release was observed with $\mathrm{POL} / \mathrm{SA} / \mathrm{V}$ matrices compared with equivalent $\mathrm{POL} / \mathrm{ET} / \mathrm{V}$ matrices.

In order to describe the mechanism of drug release the $\mathrm{n}$ values were obtained from the following well known Peppas equation [16]

$$
M_{t} / M_{\infty}=k t^{n}
$$

$M_{t}$ is the amount of the drug released at time t, $M_{\infty}$ is 


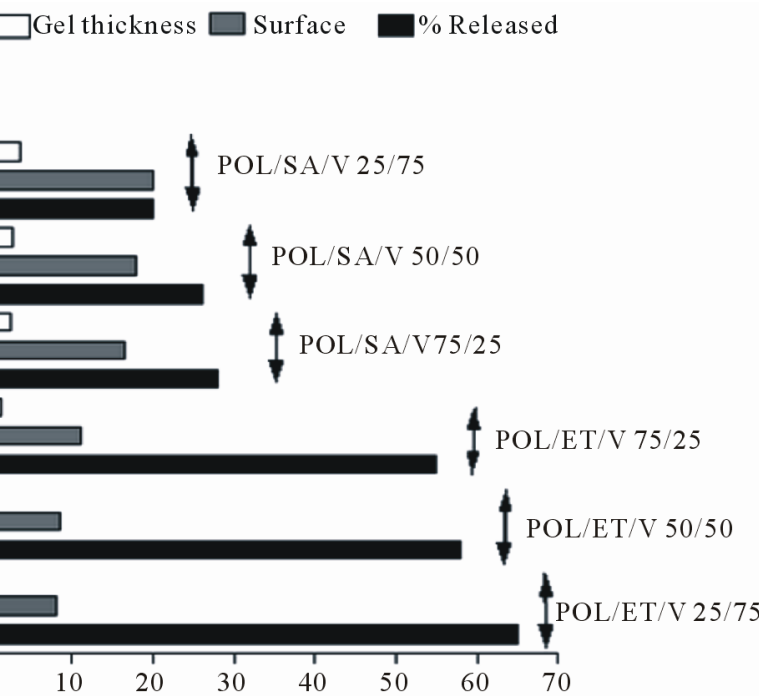

Figure 8. Relationship of drug release with surface and gel layer thickness.

the amount of drug released over a very long time that corresponds in principle to the initial loading, $\mathrm{k}$ is the kinetic constant and $\mathrm{n}$ is the diffusional exponent which depends on the release mechanism. For a cylindrical matrix values $\mathrm{n}=0.5$ indicate Fickian release, values $0.45<$ $\mathrm{n}<0.89$ indicate anomalous release kinetics (coupled diffusion/relaxation) and $0.89<\mathrm{n}<1$ indicate a zero order release also known as purely relaxation-controlled drug release.

The $n$ values for POL/SA/V 25\%/75\%, 50\%/50\% and $75 \% / 25 \%$ matrices were $0.88,0.75$ and 0.66 respectively indicating anomalous release kinetics and suggesting that the drug release was mainly governed by swelling/erosion and resulting in a rather constant release rate. On the other hand the $n$ values for POL/ET/V 25\%/75\%, 50\%/ $50 \%$ and $75 \% / 25 \%$ matrices were $0.58,0.55$ and 0.53 respectively indicating mainly diffusional drug release in combination with an erosion release mechanism.

\section{Conclusions}

An image analysis method was employed to study and evaluate the dimensional and gel evolution changes in mixture tablets of Polyox with sodium alginate or Ethocel.

The findings showed that the dimensional expansion of the examined tablets was greater for the hydrophilic mixtures POL/SA or POL/SA/V compared to POL/ET or $\mathrm{POL} / \mathrm{ET} / \mathrm{V}$. Similarly the POL/SA or POL/SA/V mixtures displayed faster and greater expansion in gel growth. Moreover as the amount of sodium alginate was larger in POL/SA tablets a greater expansion in the tablet size and gel thickness was observed. In contrast as the amount of Ethocel increased in POL/ET tablets a smaller expansion and thinner layer was observed. These findings may be attributed to the properties of sodium alginate and Ethocel. In addition these characteristics may have influenced drug release from the examined tablets.

In general our findings show that $\mathrm{POL} / \mathrm{SA} / \mathrm{V}$ matrices, due to their thicker gel layer produce a thick durable and more effective barrier, a characteristic necessary for the preparation of sustained drug release delivery systems in order to achieve sustained release delivery. For this reason a slower and smaller overall drug release was observed with $\mathrm{POL} / \mathrm{SA} / \mathrm{V}$ compared with equivalent POL/ ET/V matrices. Drug solubility appears to play a noteworthy role in the overall percentage of drug release as well as the release mechanism.

Finally it is apparent that by changing the ratio of the polymer in the mixture we may modify the percentage of drug release from these matrices.

\section{Acknowledgements}

This investigation was partially supported by a grand from University of Athens.

\section{REFERENCES}

[1] S. Tomic, S. Dimitrijevic, A. Marinkovic, S. Najman and J. Filipovic, "Synthesis and Characterization of Poly(2Hydroxyethyl Methacrylate/Itaconic Acid) Copolymeric Hydrogels," Polymer Bulletin, Vol. 63, No. 6, 2009, pp. 837-851. doi:10.1007/s00289-009-0123-2

[2] R. Dey, G. Tiwary, T. Patnaik and U. Jha, "Controlled Release of 5-Aminosalisylic Acid from New pH Responsive Polymer Derived from Tamarind Seed Polysaccharide, Acrylic Acid and Polyamidoamine," Polymer Bulletin, Vol. 66, No. 5, 2010, pp. 583-598.

[3] M. Vlachou, H. Naseef and M. Efentakis, "Utilization of Hydrophilic Swellable Polymers as Carriers for Sustained Drug Delivery from Matrices and Three Layer Tablet Systems," Current Drug Delivery, Vol. 7, No. 4, 2010, pp. 334-342. doi:10.2174/156720110793360568

[4] J. Escudero, C. Ferrero and M. Jiménez-Castellanos, “Compaction properties Drug Release Kinetics and Fronts Movement Studies from Matrices Combining Mixtures of Swellable and Inert Polymers II. Effect of HPMC with Different Degrees of Methoxyhydroxypropyl Substitution," International Journal of Pharmaceutics, Vol. 387, No. 1-2, 2010, pp. 56-64. doi:10.1016/j.ijpharm.2009.12.001

[5] M. Efentakis and M. Vlachou, "Evaluation of High Molecular Weight Poly(Oxyethylene) (POLYOX) Polymer: Studies of Flow Properties Release Rate of Furosemide and Captopril from Controlled Release Hard Gelatin Capsules," Pharmaceutical Development Technnology, Vol. 5, No. 3, 2000, pp. 339-346.

[6] A. Domb, J. Kost and D. Wiseman, "Handbook of Biodegradable Polymers" Harwood Academic Publishers, Newark, 1997. 
[7] M. Efentakis and G. Buckton, "The Effect of Erosion and Swelling on the Dissolution of Theophylline from Low and High Molecular Weight Sodium Alginate Matrices," Pharmaceutical Development Technnology, Vol. 7, 2002, pp. 69-77.

[8] S. Riyajan and J. Sakdapipanich, "Development of a Controlled Release Capsule with a Sodium Alginate Matrix, Crosslinked by Glutaraldehyde and Coated with Natural Rubber," Polymer Bulletin, Vol. 63, No. 4, 2009, pp. 609622. doi:10.1007/s00289-009-0126-Z

[9] S. Indiran Pather, I. Russel, J. Syce and S. Neau, "Sustained Release Theophylline Tablets by Direct Compression Part 1: Formulation and in Vitro Testing," International Journal Pharmaceutics, Vol. 164, No. 1-2, 1998, pp. 1-10. doi:10.1016/S0378-5173(97)00348-7

[10] R. Bettini, P. Catellani, P. Santi, G. Massimo, N. Peppas and P. Colombo, "Translocation of Drug Particles in HPMC Matrix Gel Layer: Effect of Drug Solubility and Influence on Release Rate," Journal Controlled Release Vol. 70, No. 3, 2001, pp. 383-39. doi:10.1016/S0168-3659(00)00366-7

[11] P. Colombo, R. Bettini, P. Santi, A. De Asentiis and N. Peppas, "Analysis of the Swelling and Release Mechanisms from Drug Delivery Systems with Emphasis on Drug Solubility and Water Transport," Journal Controlled Release, Vol. 39, No. 2-3, 1996, pp. 231-242. doi:10.1016/0168-3659(95)00158-1
[12] M. Efentakis, I. Pagoni, M. Vlachou and K. Avgoustakis, "Dimensional Changes, Gel Layer Evolution and Drug Release Studies in Hydrophilic Matrices Loaded with Drugs of Different Solubility," International Journal Pharmaceutics, Vol. 339, No. 1-2, 2007, pp. 66-75. doi:10.1016/j.ijpharm.2007.02.023

[13] E. Papadimitriou, G. Buckton and M. Efentakis, " Probing the Mechanism of Swelling of Hydropropylmethylcellulose Matrices," International Journal Pharmaceutics, Vol. 98, No. 1-3, 1993, pp. 57-62. doi:10.1016/0378-5173(93)90041-D

[14] M. Vlachou, H. Naseef and M. Efentakis, "Image Analysis Studies of Dimensional Changes in Swellable Hydrophilic Polymer Matrices," Polymers Advanced Technologies, Vol. 15, No. 11, 2004, pp. 683-689. doi:10.1002/pat.531

[15] M. Efentakis and K. Stamoulis, "An Investigation into the Swelling Properties, Dimensional Changes and Gel Layer Evolution in Chitosan Tablets Undergoing Hydration," Advances Polymer Technology, Vol. 28, No. 1, 2009, pp. 32-39. doi:10.1002/adv.20147

[16] R. Korsmeyer, R. Gurny, E. Doelker, P. Buri and N. Peppas, "Mechanisms of Solute Release from Porous Hydrophilic Polymers," International Journal Pharmaceutics, Vol. 15, No. 1, 1983, pp. 25-35. doi:10.1016/0378-5173(83)90064-9 